\title{
Survey of Tick-borne-disease from Ornithodoros spp. in Uninhabited Islands of Korea.
}

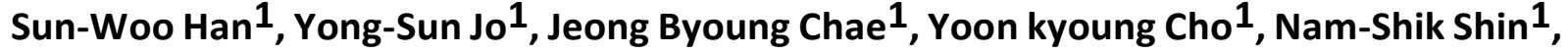 \\ Hee-Jeong Youn ${ }^{1}$, Hwa-Young Youn ${ }^{1}$, Hyang-Mi Nam ${ }^{2}$, Hyun-Joo Kim², Hae-Eun Kang ${ }^{2}$, \\ Joon-seok Chae 1 \\ ${ }^{1}$ Seoul national university, Seoul, Korea (the Republic of), ${ }^{2}$ Animal and Plant Quarantine Agency, Gimcheon, Korea (the Republic of) \\ Objective
}

The aim of this study is to survey data of ticks distribution of Korean islands and to investigate pathogens in Argasid ticks.

Introduction

Ticks and tick-borne diseases have been thought global important issues, because it's affect to animal and human health and are the cause of significant economic losses. The genus Ornithodoros spp., which is included in Family Argasidae, is usually associated with wild animals including seabirds and it was difficult to investigate because seabirds' nests are found in inaccessible uninhabited islands. However, Ornithodoros spp. has been known for the vector of many diseases including African swine fever.

\section{Method}

In this study, nest with soil and litter of seabirds were collected, to investigate Ornithodoros species from 9 uninhabited islands, Nan-do, Chilsan-do, Chilbal-do, Sogukhol-do, Googul-do, Gaerin-do, Sasu-do, Hong-do (Hallyeohaesang) and Dokdo located western and southern part of the Korea from July, 2017 to September in 2018. The islands are known for breeding places of migratory and resident birds. Maximum ten nests with soil and litter of seabirds were collected from one uninhabited island for the conservation of the islands environment. Ticks were collected from nest with soil and litter of seabirds using Tullgren funnel and were assayed for tick identification by PCR using 16S rRNA gene and tick-borne pathogens including Rickettia spp., Borrelia spp., Bartonella spp., Ehrlichia chaffensis, Ehrlichia canis, Anaplasma phagocytophilum and Anaplasma bovis by nested PCR.

\section{Results}

Total 65 Ornithodoros species ticks from 338 seabird's (black-tailed gull, Larus crassirotris; streaked shearwater, Calonectris leucomelas and Swinhoe's storm petrel, Oceanodroma monorhis) nesting soil with litter in 9 uninhabited islands. In the sequence identification of 16S rRNA gene fragment of Ornithodoros species, O. capensis and O. sawaii were 37 and 28 , respectively. In the analyses of tick-borne pathogens, Borrelia spp. (n=5) was detected in $O$. sawaii from Gaerin-do and Googul-do. The total detection rate of Borrelia sp. from Ornithodoros spp. was $7.69 \%$ (5/65).

\section{Conclusions}

In this research, we discovered that $O$. sawaii are habitat in west and southern part of uninhabited islands to breeding place of black-tailed gulls and streaked shearwater and $O$. capensis are habitat in uninhabited islands related to breeding place of streaked shearwater. This is first report of the Borrelia spp. from Ornithodoros sawaii in Korea.

\section{Acknowledgement}

This research was supported by a fund (no. Z-1543085-2017-18-01) from Research of Animal and Plant Quarantine Agency, the Republic of Korea. 